\title{
Asteroids' spin axis distribution
}

\author{
A. Marciniak and T. Michałowski
}

\author{
Astronomical Observatory, Adam Mickiewicz University, Słoneczna 36, 60-286 Poznań, Poland \\ e-mail: aniab@lab.astro.amu.edu.pl
}

Received 18 November 2009 / Accepted 22 December 2009

\section{ABSTRACT}

\begin{abstract}
Analysing the database of asteroid models from inversion techniques (DAMIT) ${ }^{\star}$, we found that the spin axis of asteroids do not avoid the ecliptic plane, which had been widely understood since the early days of asteroid physical studies. Previously observed depopulation was probably caused by the EAM methods bias, which is absent if the asteroid shapes are modelled as more complex rather than triaxial ellipsoids.
\end{abstract}

Key words. techniques: photometric - minor planets, asteroids: general

\section{Introduction}

Two decades ago, approximately 20 asteroids had known spin and shape parameters (such as sidereal period of rotation, spin axis position, and shape model axis ratio). This small sample already exhibited a nonrandom distribution of spin axis latitudes. Zappalà \& Kneževic̀ (1984) and Barucci et al. (1986) noted a clustering of spin-vector positions at intermediate ecliptic latitudes, which was later found to be partly caused by the methods used for modelling. A similar picture emerged from the studies of Drummond et al. (1988), and Magnusson \& Lagerkvist (1990). The distribution of spin axes was bimodal, some asteroids rotating in a retrograde sense, some in a prograde sense, and almost none having axes close to the ecliptic plane. There was also some excess of asteroids with prograde rotation. These results were surprising in the light of accepted theory for the collisional history of the main asteroid belt, which should leave randomly oriented spin axes, perhaps with a small excess of prograde rotating objects.

Magnusson (1986) speculated about the possible causes the gap near the ecliptic plane. The solution clouds generated by the amplitude-magnitude methods were artificially separated by the ecliptic plane. Thus the $\beta_{\mathrm{p}}$ values were shifted towards higher latitudes in both hemispheres. Another possibile cause of the ecliptic gap was noted by Magnusson et al. (1989). The epoch method could have introduced a bias because of its inability to use the near-zero amplitude lightcurves often exhibited by "low-pole" objects. These methods had problems finding the lightcurve extrema needed for the timing. Drummond et al. (1988) considered the gap to be an observational selection effect, favouring large amplitude objects, which must have spin axes that are placed far from the ecliptic plane.

At this early stage of research, Binzel (1988) found that large amplitudes of brightness variations exhibited by all of the Koronis family members might imply possible grouping of their spin axes. In another work, Binzel (1990) indeed found groupings of these asteroids' pole longitudes. This was later

\footnotetext{
* The DAMIT database is described in detail in Ďurech et al. (2010).
}

spectacularly confirmed by Slivan (2002), who identified not only spin axis but also spin period clusters among this family of asteroids. This clustering can be most accurately explained by the thermal recoil YORP effect (Vokrouhlický et al. 2003). To date, the Koronis family was the only one studied in that way, but the effect may also be present in other asteroid families.

Another paper on the subject of the spin axis distribution is the work of Pravec et al. (2002), showing a very asymmetric distribution of asteroids' spin axis latitudes. The work of Kryszczyńska et al. (2007) collates all reliable asteroid spin axes determinations from various techniques. It was based on the database of asteroid models developed by Per Magnusson (1989) and presently maintained at Poznań Observatory (Poland) ${ }^{1}$. The asteroid models (92 objects) analysed in their paper are only the most reliable ones, based on extensive datasets and refined pole determination algorithms, mostly EAM methods. However, the distribution of ecliptic latitudes of asteroid poles remained even more pronouncedly bimodal, with a deep gap near the ecliptic plane. As the authors claimed, the observational bias towards high amplitude objects should be lower for the larger sample of objects observed with modern photometric techniques. No physical phenomenon was found to explain this depopulation of spin vectors close to the ecliptic plane, which therefore remained a mystery.

\section{Models from lightcurve inversion}

Since the lightcurve inversion method was introduced (Kaasalainen \& Torppa 2001; and Kaasalainen et al. 2001), over 100 asteroids have been modelled with this technique. The method is a major breakthrough in the modelling of asteroidal spin and shape parameters. It directly uses all datapoints from the available lightcurves and makes no assumptions about the object's shape. Thus interesting and complex shapes were discovered. It became possible to fit the lightcurves with triple extrema and other irregularities or even attempt nonconvex shape models. Using the classical EAM methods, this was

1 wWW.astro.amu.edu.pl/Science/Asteroids 


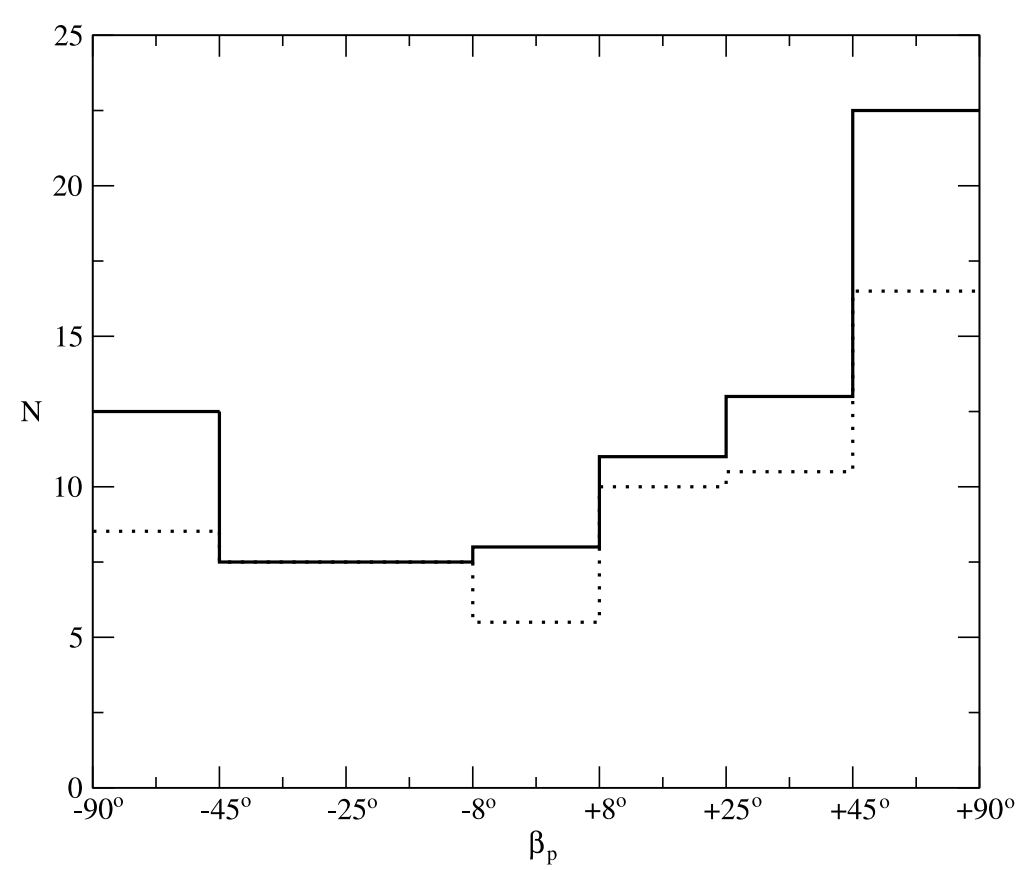

Fig. 1. Histogram containing ecliptic latitudes of spin axis of all reliable asteroid models obtained with the lightcurve inversion method. Bins are chosen so as to represent similar surfaces on the $\lambda, \beta$ sphere. The dotted line represents robust solutions.

impossible, because their principle assumption was the ellipsoidal shape of an asteroid. The errors in spin axis coordinates determined by the lightcurve inversion method are also usually limited to $\pm 5^{\circ}$, ensuring the method to be the most precise of the methods in use.

Asteroid models created using the lightcurve inversion method are collected by Josef Durech in the DAMIT database ${ }^{2}$, described in Ďurech et al. (2010). Our research group from Poznań has contributed to that database as authors of both observations and models (i.e., Michałowski et al. 2004, 2005, 2006; Marciniak et al. 2007, 2008, 2009a,b; Durech et al. 2007).

As the sample of objects modelled by this technique became significant, one could perform statistical investigations. Torppa et al. (2008) parametrized the shape models and found correlations both between shapes and rotational periods and between shapes and sizes. We were interested in the distribution of mainbelt asteroids spin axes in space. This distribution is shown in Fig. 1, which shows only the spin axis ecliptic latitudes as we did not find any specific trends in the distribution of longitudes. The histogram contains all the reliable asteroid poles obtained with lightcurve inversion on full lightcurves ( 82 objects in all, DAMIT state for 6 November 2009). Where there was a mirror solution, both pole latitudes were taken each with the weight $1 / 2$. The bins are identical to those in the paper by Kryszczyńska et al. (2007), representing similar areas on the celestial sphere. The dotted line represents only the most certain solutions. The remaining solutions are considered "preliminary", although this notion most often refers only to the longitude of the spin axis. In the above-mentioned paper, asteroid spin axes were also referred to their orbital planes, but the distribution of spin axis latitudes remained very similar. Thus we refer the spin axes to the ecliptic plane only.

2 Database of Asteroid Models from Inversion Techniques, available at: http://astro.troja.mff.cuni.cz/projects/asteroids3D

\section{Results}

In contrast to previous findings, there seems to be no gap in the pole distribution close to the ecliptic plane. This gap is partially filled with asteroids whose poles were placed at higher latitudes by previous modelling. With larger datasets, and more importantly, more complex shapes being allowed, these objects were inferred to have substantially "lower" poles, possibly because of the photometric behaviour of the ellipsoidal shapes. To create a triaxial ellipsoid that viewed pole-on exhibits significant light variations, one must substantially lift its pole away from the ecliptic plane. A complex shape can exhibit changes in reflected sunlight even when the angle between its spin axis and viewing direction is very small. But even flat lightcurves are not a problem for the inversion method, when there are also other lightcurves in the dataset.

The effect of artificially increasing $\left|\beta_{\mathrm{p}}\right|$ values away from the ecliptic plane calculated by EAM methods can be partially responsible for the ecliptic gap in the database of asteroid spins inferred by all methods (Kryszczyńska et al. 2007) in yet another way. The final spin-axis coordinates used in their analysis are often weighted averages from various solutions. Thus, even when the latest pole solution from the inversion method placed an asteroid's pole close to the ecliptic plane, this averaging increased it to higher ecliptic latitudes in both hemispheres.

Another surprise concerning the new distribution is that the number of prograde solutions is almost double that of the retrograde ones (46 to 27 , respectively, omitting the middle, "ecliptic" bin). The absence of the ecliptic gap makes the pole distribution consistent with the predictions of asteroid evolution theories, but this large excess of prograde rotators remains a mystery. One possible explanation is that it might be produced by YORP-caused spin clusters in asteroid families, as for the Koronis family, present in the sample investigated here (Slivan et al. 2003). Members of this family are mostly found in the two 
most extreme peaks in Fig. 1. If one excludes all but two representative members of both spin clusters in the Koronis family, the leftmost peak is lowered by a factor of 4 and the two rightmost peaks attain marginally smaller heights than in the original histogram. As for other dynamical families or clusters, there are only a few Flora family members, but each resides in a different peak. Significantly more data are needed to find correlations in other dynamical groups.

As the spin clustering in asteroid families cannot explain the large excess of prograde rotators, one has to search for a different mechanism. If the Yarkovsky drift of retrograde rotators towards the Sun was efficient here, it should have created the opposite distribution of more retrograde rotators being represented in this sample, because they would have been dragged towards the inner main-belt, thus observed more easily. A partial problem with both theories is the size of most of the objects, as the majority of them have diameters of around $100 \mathrm{~km}$, with a relatively small number having diameters as small as $20 \mathrm{~km}$. The thermal recoil force works efficiently on objects smaller than $40 \mathrm{~km}$ (Vokrouhlický et al. 2003).

\section{Conclusions}

The dataset of asteroids that have been modelled with the lightcurve inversion method definitely contains a mixture of physically various object. But the main advantage of this collection is that all objects have been modelled with the same technique, unlike previous investigations. With more objects having been modelled, especially of small size, and also slow rotating ones, it will be possible to make divisions between them and draw conclusions about the statistics of various groups of asteroids.
Acknowledgements. DAMIT database is maintained and made available by Josef Ďurech, at http://astro.troja.mff.cuni.cz/projects/ asteroids3D. This work was supported by grant N N203 302535 from the Polish Ministry of Science and Higher Education.

\section{References}

Barucci, M. A., Bockelee-Morvan, D., Brahic, A., et al. 1986, A\&A, 163, 261 Binzel, R. P. 1988, Icarus, 73, 303

Binzel, R. P. 1990, in Asteroids, Comets, Meteors III, ed. C.-I. Lagerkvist, H. Rickman, B. A. Lindblad, \& M. Lindren, Uppsala Universitet, 15

Drummond, J. D., Weidenschilling, S. J., Chapman, C. R., \& Davis, D. R. 1988, Icarus, 76, 19

Ďurech, J., Kaasalainen, M., Marciniak A., et al. 2007, A\&A, 465, 331

Durech, J., Sidorin, V., \& Kaasalainen, M., 2010, A\&A, in press

Kaasalainen, M., \& Torppa, J. 2001, Icarus, 153, 24

Kaasalainen, M., Torrpa, J., \& Muinonen, K. 2001, Icarus, 153, 37

Kryszczyńska, A., La Spina, A., Paolicchi, P., et al. 2007, Icarus, 192, 223

Magnusson, P. 1986, Icarus, 68, 1

Magnusson, P. 1989, in Asteroids II, ed. R. P. Binzel, T. Gehrels, \& M. S. Matthews (Tucson: Univ. of Arizona Press), 1180

Magnusson, P., \& Lagerkvist, C.-I. 1990, A\&AS, 86, 45

Magnusson, P., Barucci, M. A., Drummond, J. D., et al. 1989, in Asteroids II, ed. R. P. Binzel, T. Gehrels, \& M. S. Matthews (Tucson: Univ. of Arizona Press), 67

Marciniak, A., Michałowski, T., Kaasalainen, M., et al. 2007, A\&A, 473, 633

Marciniak, A., Michałowski, T., Kaasalainen, M., et al. 2008, A\&A, 478, 559

Marciniak, A., Michałowski, T., Hirsch, R., et al. 2009a, A\&A, 498, 313

Marciniak, A., Michałowski, T., Hirsch, R., et al. 2009b, A\&A, 508, 1503

Michałowski, T., Kaasalainen, M., Marciniak, A., et al. 2005, A\&A, 443, 329

Michałowski, T., Kaasalainen, M., Polińska, M., et al. 2006, A\&A, 459, 663

Michałowski, T., Kwiatkowski, T., Kaasalainen, M., et al. 2004, A\&A, 416, 353 Pravec, P., Harris, A. W., \& Michałowski, T. 2002, in Asteroids III, ed. W. Bottke,

A. Cellino, P. Paolicchi, \& R. P. Binzel (Tucson: Univ. of Arizona Press), 113 Slivan, S. 2002, Nature, 419, 49

Slivan, S., Binzel, R., da Silva, L., et al. 2003, Icarus, 162, 285

Torppa, J., Hentunen, V.-P., Pääkkönen, P., et al. 2008, Icarus, 198, 91

Vokrouhlický, D., Nesvorný, D., \& Bottke, W. 2003, Nature, 425, 147

Zappalà, V., \& Kneževic̀, Z. 1984, Icarus, 59, 435 